\title{
Versorgungsgesetz 2011 - Die Zukunft der Versorgung?
}

Karl-Heinz Schönbach

Karl-Heinz Schönbach ist

Geschäftsführer des Geschäftsbereichs Versorgung beim AOK-

Bundesverband.

\section{Der Bundesminister für Gesundheit hat Anfang April} 2011 die Eckpunkte der Koalition für das Gesetzgebungsverfahren zum Versorgungsgesetz 2011 vorgestellt. Ende Mai wird es den Referentenentwurf geben. Erwarten wir damit die Zukunft der Versorgung? Wohl nicht, und der Minister, dem mit der Finanzierungsreform nur Stückwerk vergönnt war, vermied nun alles Hochtrabende, bevor er als Vizekanzler zu neuen Aufgaben eilte. Wohltuend für seinen Nachfolger, dass eine Jahrhundertreform erst gar nicht angekündigt wurde. Gleichwohl wird man sich Diagnose, Ziele und Instrumente der politischen Eckpunkte anschauen müssen, um ihr Potential auszuloten.

\section{Ausgangslage}

Nachdem noch die Große Koalition das Ende der Kostendämpfung eingeläutet und den Ärzten Mehreinnahmen in Milliardenhöhe beschert hatte, wendet sich die schwarz-gelbe Koalition nun den Ärzten als Versorger zu. Zusätzliche Finanzmittel von mehr als 5 Mrd. Euro scheinen zuvor mehr oder weniger spurlos versickert zu sein. Das Vorhaben war Ende 2010 nicht ohne Grund als »Versorgergesetz « angekündigt worden, bis der gesundheitspolitische Sprecher der Unionsfraktion, Jens Spahn, reklamierte, für die Versicherten, die Milliarden zusätzlich aufgebracht hätten, müsse nun auch eine bessere Versorgung erkennbar werden. Gleichwohl stellen die vereinbarten Eckpunkte nun dominant auf das Angebot und die Verteilung von Ärzten in der Zukunft ab, während aktuelle Verbesserungen für die Patienten unter ferner Liefen landen. Allerdings wendet der Gesundheitsminister bei der For- mulierung der Reformeckpunkte große Sorgfalt auf, der Kampagne der verfassten Ärzteschaft um einen so genannten » Ärztemangel « nicht allzu offensichtlich aufzusitzen. Gleichwohl: trotz der seit den Zeiten der allseits beklagten Ärzteschwemme »nach wie vor steigenden Arztzahlen " stünden »nicht mehr in allen Bereichen und Regionen Ärztinnen und Ärzte ausreichender Zahl zur Verfügung « und immerhin seien »Trends erkennbar, nach denen sich der drohende Arztmangel zumindest in einer frühen Phase « befände und "mittelfristig zu verstärken drohe«. Selten hat sich Politik mit solcher Inbrunst seismographischer Chiffren bedient, der Feminisierung eines Berufsstandes nachgesonnen und sich über eine veränderte »work-life-balance« gebeugt.

Rüttelt der Chronist die Worte durch, bleibt ein Verteilungsproblem: Die Kassenärztlichen Vereinigungen sind als Körperschaften des öffentlichen Rechts nicht in der Lage, ein 
flächendeckendes Angebot sicherzustellen: Es fehlen Allgemeinmediziner insbesondere in strukturschwachen Gebieten, auf dem Lande und in den Städten - dort, wo der PKV-Anteil gering ist. Dagegen haben wir insbesondere in den Zentren ein Überangebot an Fachärzten. Diese Diagnose lässt der Gesundheitsminister zwar erahnen klar ausgesprochen wird sie nicht. Zudem bleibt vollends im Dunkeln, wie aktuell aus einem Überangebot an Fachärzten gleichwohl subjektiv die Anmutung einer Art Ärztemangel resultieren kann. Wartezeiten der Versicherten sind ja unverkennbar. Dabei zeigt die Kostenstrukturerhebung des Statistischen Bundesamtes, dass bei einem Versichertenanteil der GKV von rund $87 \%$ etwa bei den Hautärzten gerade noch $53 \%$ der Umsätze auf die GKV entfallen, bei den Chirurgen 62 $\%$, den Urologen $62 \%$ und den Augenärzten $63 \%$. Mehr als einem Drittel der GKV-Versicherten werden inzwischen in der Arztpraxis privatärztliche Zusatzleistungen offeriert. Und einige Fachärzte haben die Sprechstundenzeiten mittlerweile der Peripherie des vertragsärztlich gerade noch Zulässigen genähert. Da das Vergütungssystem einen relativ hohen Grad der Pauschalierung aufweist, werden Patienten in hoher Zahl im Praxiskreislauf gehalten, ohne dass Behandlungsinhalte und Ziele subjektiv den Eindruck einer dem jeweiligen Patientenproblem angemessenen Versorgung erkennen lassen.

Die Angebotsfragen hinter den Ärztezahlen bleiben dem Gesundheitsminister offenbar verborgen. Er postuliert kraftvoll: »An dem Ziel einer flächendeckenden medizinischen Versorgung in Deutschland wird festgehalten. « Dies sei angesichts des (dennoch?) drohenden Arztmangels eine "große Herausforderung «. Und von daher bedürfe es der Weiterentwicklung der Bedarfsplanung. Leitidee der Überlegungen und Vorschläge sei die Verbesserung bzw. der Erhalt der freiberuflichen Ausübung des Arztberufes und der Diagnose- und Therapiefreiheit. Angesprochen werden dann noch Bürokratieabbau und "Effizienzreserven an der Sektorengrenze«. Man kann das als Gedöns bei einer an sich pragmatisch angelegten Reform abtun und den Mantel des Schweigens darüber breiten. Aber das mit den Eckpunkten an- gebotene konzeptionelle Fundament der Koalition ist nicht erkennbar. Umso ärgerlicher sind redaktionelle Reste an Pathos (»Erst eine solche Reform ergänzt die zurückliegende Finanzierungsreform zu einem Gesamtkonzept. «), mit denen der Minister inzwischen doch spart.

\section{Weiterentwicklung der Bedarfsplanung}

Die Reformeckpunkte greifen die gesundheitspolitische Diskussion um eine »Flexibilisierung « der Bedarfsplanung auf, nach der die Planungsräume differenziert nach dem Grad der Spezialisierung der ärztlichen Versorgung und der mit ihm abnehmenden Wohnortnähe festgelegt werden sollen. Die künftige Justierung des Systems nach Verhältniszahlen soll aufgrund sachgerechter Kriterien unter Berücksichtigung der demografischen Entwicklung und nicht länger stichtagsbezogen erfolgen. Rätsel gibt der nicht weiter definierte Begriff der "strukturschwachen « Gebiete auf. Offenbar insbesondere aufgrund der mit den Ländern teils noch strittigen Beteiligung an der ambulanten Bedarfsplanung wird dem Gemeinsamen Bundesausschuss (G-BA) für die Weiterentwicklung ein großer Entscheidungsspielraum hinsichtlich der Gestaltungsmöglichkeiten vorbehalten. Damit wird das Reformkonzept noch nicht wirklich umrissen. Die Entscheidungsprozesse für die tatsächliche Ausgestaltung der Bedarfsplanung werden in die Selbstverwaltung verlagert. Somit könnte im G-BA ebenso das Konzept der Krankenkassen wie das Konzept der Kassenärztlichen Bundesvereinigung - die kleinräumige Bedarfsplanung - wieder auf die Tagesordnung gesetzt werden. Die Differenzierung der Planungsräume nach versorgungsinhaltlichen Stufen (hausärztliche Versorgung, fachärztliche Versorgung und spezialisierte Leistungen) wird lediglich als »möglich « eingeräumt, nicht aber als Prinzip vorgegeben.

Wenn die offenen Fragen zwischen Bund und Ländern geklärt sind, sollte die Gesetzgebung einen regionalen Bedarfsplanungsansatz klarstellen. Dabei schafft der G-BA durch bundesweit geltender Kriterien die Grundlage, die auf Landesebene hinsichtlich der Definition der konkreten Festlegung der Räume für die jeweiligen Versorgungsstufen und der Feinjustierung ausge- füllt wird. In diesem Falle ist es nicht erforderlich, »regionale Bedarfe « als Ausnahme von den G-BA-Regeln wieder zu beleben.

Offenbar aus den gleichen Gründen verschenkt der Gesundheitsminister in diesem Schritt die Chance, zu einer sektorübergreifenden ambulanten Bedarfsplanung zu kommen. Einem Gremium, das die Länder dazu künftig einrichten können sollen, werden keinerlei Befugnisse zugemessen. Diesen Schritt müsste er aber gehen, um bei Vorstößen in neue sektorunabhängige Versorgungsformen, wie der »spezialärztlichen Versorgung ", nicht im ordnungspolitischen Niemandsland zu enden, wo es weder Bedarfsplanung, noch schlüssig gestufte Vertragssysteme gibt.

Der Gesundheitsminister skizziert Eckpunkte für ein Konzept einer neu zu entwickelnden ambulanten spezialärztlichen Versorgung, die sektorunabhängig von Spezialistenteams in Arztpraxen oder Krankenhäusern erbracht werden kann. Adressiert werden Leistungen für Erkrankungen mit besonderen Krankheitsverläufen, seltenen Erkrankungen, hochspezialisierten Leistungen und bestimmten ambulanten Operationen sowie stationsersetzenden Eingriffen. Die Konkretisierung der Leistungen sowie der Anforderungen an die Leistungserbringung und Qualitätssicherung soll dem G-BA vorbehalten sein. Die Leistungen erbringen kann dann auf Grundlage des EBM »wer will « mit »freiem « Zugang (zur Abrechung bei den Krankenkassen). Das Konzept ist weder mit der gerade neu konzipierten ambulanten Bedarfsplanung verbunden, noch in das Vertragssystem eingepasst. Dazu könnte der Gesundheitsminister folgenden Weg einschlagen:

- Unterscheidung der persönlichen Beteiligung von Krankenhausärzten an der ambulanten Versorgung mit der Folge der Zulassung durch den Landesausschuss von einer institutionellen Beteiligung des Krankenhauses bei sektorunabhängigen ambulanten

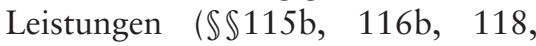
119, 120 SGB V) und Sonderformen ( $\int \mathbb{S} 140 b, 116 b$ Abs. 1, 117). Die Regelungsperspektive von »Prä/Post « und »teilstationär« sollte im Krankenhausrecht gefunden werden.

- Schrittweise Neuregelung der »sektorunabhängigen « Leistungen ambu- 
lant und stationär beginnend mit dem Ambulanten Operieren und den Spezialärztlichen Leistungen (enge Definition des BMG) unter Einbezug in eine neu aufgestellte ambulante Bedarfsplanung, bei der die $\mathrm{KH}$ unter der bestehenden Parität im Länderausschuss nach $\mathbb{\int} 90$ SGB V bei sektorunabhängigen Entscheidungen einbezogen werden. Kein »Wer will, der kann «.

- Gestuftes Vertragsmodell mit einheitlicher und gemeinsamer und selektiver Ebene plus Out-Option der Krankenkasse in einem Planungsbezirk.

Bis jetzt aber wird die Beteiligung der Krankenhäuser nicht etwa an der Trennlinie zwischen persönlicher Beteiligung des Krankenhausarztes und institutioneller Beteiligung des Krankenhauses grundlegend neu gedacht und die Krankenhausseite in die ambulante Planung einbezogen. Offerten dazu lagen dem BMG aus der Breite der Selbstverwaltung vor. Nein, die bisherige ambulante Bedarfsplanung von Ärzten und Krankenkassen soll offenbar ohne Beteiligung der Krankenhausseite die beteiligten Krankenhausärzte lediglich »erfassen «. Es werden auch nicht alle Arztgruppen bzw. spezialisierten Ärzte und Teilgebietsbezeichnungen einbezogen, was ohne Not die Baustelle der Sonderbedarfszulassungen offen hält.

\section{Beteiligung der Länder}

In ausgiebigen Konferenzen haben Bund- und Ländergesundheitsministerien die Beteiligung der Länder neu konzipiert. Die Länder, für die in der Krankenhausplanung gemeinhin keine Kränze geflochten werden, wollen an dieser Stelle Terrain gewinnen, und sie stehen oftmals unter Druck der Bürgermeister in ländlichen Regionen. Herausgekommen ist ein Konzept, das die Beteiligung der Länder ohne Schaden für ihre Aufsichtsfunktion regeln soll: Für die Arbeit an der Weiterentwicklung der Bedarfsplanungsrichtlinien im G-BA sollen die Länder ähnlich der Beteiligung der Patientenvertreter ein Mitberatungsrecht erhalten. Für den Landesausschuss zur Bedarfsplanung werden die Länder gestellt wie der BMG zum G-BA. Die Länder können an den Sitzungen teilnehmen, haben die Rechtsaufsicht, können den
Bedarfsplan aus rechtlichen Gründen beanstanden und haben nach Fristablauf ein Ersatzvornahmerecht. Ein von den Ländern gefordertes, noch weiter gehendes Initiativrecht dem Landesausschuss gegenüber hätte die Funktion der Rechtsaufsicht durchbrochen. Dass der Bund diese Hürde innerhalb einer Krankenversicherung nach Bundesrecht mit einem bundesweiten Gesundheitsfonds nicht nehmen kann, liegt auf der Hand. Ebenso quer zur rechtlichen Architektur der GKV bewegen sich die Forderungen der Länder nach einem Initiativ- und Beanstandungsrecht bei wettbewerblichen Verträgen der Krankenkassen oder der Einrichtung ihnen berichtspflichtiger Landesbeauftragter auch bundesunmittelbarer, nicht ihrer Aufsicht unterstehender Krankenkassen. Am Ende beklagen die Länder, dass bisher »Morbidität» noch nicht als gesetzlich zu berücksichtigendes Kriterium für die Bedarfsplanung nominiert wurde. Auch wenn dieses Kriterium kaum operationalisierbar anzuwenden ist, verspricht es den Ländern doch Zugang zu den Daten der Selbstverwaltung. Von daher scheint die Inszenierung eines "Streits zwischen Bund und Ländern « an dieser Stelle eher als ein "Sturm im Wasserglas « als ein »Schwarzer Freitag «, wie ein sonst regelmäßig gut unterrichteter Branchendienst textete. Und schließlich wäre es für den Gesundheitsminister taktisch unklug, allen Länderwünschen bereits in dieser Frühphase des Verfahrens nachzukommen.

\section{Niederlassungssteuerung}

Was immer auch gerechnet und geplant wird, ohne die Bewegung real existierender Ärzte bleibt es bei Luftbuchungen. Daher werden langfristig wirksame Maßnahmen adressiert, wie die Erhöhung der Studienplatzzahlen und ein Ausbau von Ausbildungskapazitäten in der Allgemeinmedizin. Die Bedingungen, unter denen Ärzte ihre Niederlassung flexibel gestalten können, sollen verbessert werden. Schon das Vertragsarztrechtsänderungsgesetz der großen Koalition hat hier viele Türen geöffnet. In zahlreichen Einzelmaßnahmen folgt die aktuelle Koalition dem nach. Dabei unterstreicht sie durch fördernde Maßnahmen positiv das Ziel, Ärztinnen die Berufstätigkeit auch als Mutter zu erleichtern.
Die entscheidende Frage für eine Umsteuerung ist aber der Abbau von Überversorgung. Dazu soll nunmehr auch Ärzten, die das 62. Lebensjahr noch nicht vollendet haben, die Abgabe der Zulassung erleichtert werden. Wie bisher sind dazu Entschädigungszahlungen aus Mitteln der Kassenärztlichen Vereinigungen (KVen) zu finanzieren. Unter diesem Vorzeichen kann sicherlich auch das Vorkaufsrecht der KVen bei der Ausschreibung von Praxen in überversorgten Regionen positiv bewertet werden. Als zweifellos nicht beabsichtigter, aber keineswegs zu unterschätzender Anstoß für einen ordnungspolitischen Durchbruch kann die neue Option gewertet werden, Zulassungen in offenen Planungsbereichen mit einem Versorgungsgrad ab 100\% zeitlich zu befristen. So kleinmütig die Rücknahme der gesetzlichen Bestimmungen für $\mathrm{Zu}$ - und Abschlage bei der Vergütung (Orientierungswert) nach dem Grad der Unter- und Überversorgung wirken mag - diese Modell der "pretialen « Steuerung hatte die Ärzteschaft selbst in die Diskussion gebracht - so eindeutig wirksam scheint der Vorstoß im Hinblick auf die befristete Zulassung in überversorgten Gebieten: Welcher Arzt wird nicht die unbefristete Zulassung in unterversorgten Bezirken mit der befristeten Zulassung in überversorgten Bezirken vergleichen? Es ist von daher auch nicht unangemessen, von der Abstaffelung der Regelleistungsvolumen in unterversorgten Bezirken abzusehen. Der Bewertungsausschuss auf Bundesebene hatte dies den Gesamtvertragspartnern auf Landesebene ohnehin bereits freigestellt, wenn ein Arzt dort überproportional viele Patienten versorgen muss. Das Abstaffelungsverbot der Vergütung in unterversorgten Gebieten betrifft zudem nur 37 Ärzte in der gesamten Republik - soviel zum Ärztemangel! Weitergehende preisliche Steuerungsmaßnahmen und die Handhabung von Strukturfonds aus der Kollektivvergütung sollten jedoch zwingend der vertraglichen Vereinbarung vor Ort vorbehalten werden.

Weitere Maßnahmen, um das Angebot an Ärzten auf dem Lande zu verbessern, sind die Ermächtigung von Ärzten aus Rehabilitations- und Pflegeeinrichtungen bei lokalem Versorgungsbedarf, die Lockerung der Resi- 
denzpflicht, die Förderung mobiler Versorgungskonzepte, der Telemedizin sowie der Delegation und Substitution ärztlicher Leistungen auf der Grundlage von Vereinbarungen in den Bundesmantelverträgen.

Es bleibt aber der Umstand, dass Ärzte sich dort niederlassen, wo sie studiert haben, die Oper lockt und ein möglichst hoher PKV-Anteil. Dass diese Merkmale nicht streng mit dem Versorgungsbedarf korrelieren, ist ein ordnungspolitischer Fauxpas. Es macht aber keinen Sinn, darüber hinweg zu sehen und aktionistisch gegenzusteuern. Was zählt ist die Ökonomie, daher wird man die Botschaft auf einen Geldschein schreiben müssen. Will die Koalition somit politisch durchsetzen, dass Ärzte für 87 Prozent der Bevölkerung in der GKV dort arbeiten, wo sie benötigt werden, wird sie nur konsequent von einem korporatistischen (Verteilungs-) System abrücken können,

- in dem sich heute jeder Arzt gleich welcher Fachrichtung als Zwangsmitglied der Kassenärztlichen Vereinigung niederlassen kann

- und die Kassenärztliche Vereinigung überversorgte Gebiete für neue Niederlassungen zwar sperren, überzählige Sitze aber nicht vom Markt nehmen kann, weil solche Sitze (Lizenzen), deren Wert in der öffentlichrechtlichen Planung begründet ist, rechtlich zu einem privaten Eigentum mutieren.

Gerade aus Sicht einer liberalen Koalition würde der Staat demgegenüber seiner Aufgabe, die medizinische Daseinsvorsorge $\mathrm{zu}$ organisieren, versorgungsorientiert und ordnungspolitisch freiheitlich dadurch ausfüllen, dass er den Krankenkassen regional strukturiert eine hinreichende Vergabe von Lizenzen auferlegte. Diese Diskussion hat die Koalition nun immerhin mit »zeitlich befristeten Zulassungen in überversorgten Gebieten« eröffnet.

\section{Entwicklung der Kassenärztlichen Vereinigungen und MVZ}

Darüber hinaus setzt die Koalition besonders auf die Kassenärztlichen Vereinigungen. Sie sollen ähnliche Rechte zur organisatorischen Weiterentwicklung und Fusion erhalten wie die Krankenkassen. Anders als die Krankenkas- sen sollen sie in unterversorgten Bezirken Eigeneinrichtungen bilden können. Eigeneinrichtungen der Kommunen sind demgegenüber nachrangig gestellt. Die Gründung Medizinischer Versorgungszentren, die im Kreis der Mitglieder der KVen zu einer höheren Konzentration führen könnten, wird auf Vertragsärzte und Krankenhäuser beschränkt. Die Rechtsformen der MVZ werden auf Personengesellschaften und GmbHs beschränkt. Die Form der Aktiengesellschaft soll für Neugründungen ausgeschlossen werden. Die Leitung in der medizinischen Versorgung des MVZ soll "rechtlich und faktisch " in ärztlicher Hand liegen. Auch sollen Vertragsärzte künftig bei frei werdenden Arztsitzen ein Vorkaufsrecht gegenüber MVZs haben und ein Vertragsarztsitze nur dann in ein MVZ verlegt werden können, wenn dem keine Versorgungsgesichtspunkte entgegenstehen.

\section{Honorarreform}

Eine in hohem Maße kryptische Baustelle des Versorgungsgesetzes bleibt die Honorarreform für die niedergelassenen Ärzte. Hatte die Koalition sich im Rahmen des GKV-Finanzierungsgesetzes noch auf euphemistische Ankündigungen $\mathrm{zu}$ einem »einfachen und gerechten Honorarsystem « beschränkt und den Bewertungsausschuss bis April 2010 um einen Bericht gebeten, wird in den Eckpunkten nun die »Regionalisierung « der Honorarverteilung nachgelegt, ohne sie in ein erkennbares Konzept einzubetten. In der Tat spricht viel dafür, den Bewertungsausschuss auf Bundesebene von allzu irrlichternden Eingriffen in die Honorarverteilung abzuhalten. Wer die Honorarverteilung allerdings auch auf KV-Ebene vom Einvernehmen mit den Krankenkassen trennt - und das will die Koalition hat zumindest nicht erkannt, welches Diskriminierungspotential hiervon für kleine Arztgruppen oder etwa selektive Verträge der Krankenkassen ausgeht. Nicht, dass die Krankenkassen den Überlegungen der KV bei der Honorarverteilung generell refraktär gegenüberstünden und ein "Benehmensregelung « diese Blockade lösen müsste: Nein, ein gesetzlicher Bedarf besteht in keiner Weise. Die KVen beanspruchen vielmehr "Autonomie « bei der Honorarverteilung, um mit den Verteilungswir- kungen von Selektivverträgen allein die »Selektivärzte « belasten zu können. Die Bereinigung von Honorar aus der Gesamtvergütung ist kein Problem, wenn die Honorarverteilung jegliche Umsteuerung unterbindet.

Verbindet sich die Autonomie bei der Honorarverteilung, die jeder marktwirtschaftlichen Allokation Hohn spricht, mit dem diagnosebezogenen Aufteilungsmodell der Gesamtvergütungen auf die Kassenärztlichen Vereinigungen, lieferte der Gesundheitsminister die Beitrags- und Steuerzahler einem komplett autokratischen System der Kassenärztlichen Vereinigungen aus. Verträge auf Landesebene spielten keine Rolle mehr, wenn die zu verteilenden Mittel - unabhängig von der Versorgungsstruktur ambulant und stationär sowie unabhängig von den tatsächlichen Leistungen der Vertragsärzte - den KVen nach Diagnoselasten zugemessen und von den Krankenkassen nach Anteilen an den Diagnoselasten finanziert würden. Die KVen könnten dann mit Diagnosen Einnahmen schreiben und sie autonom verteilen, während die Krankenkassen für Risiken Zahlungen zu leisten hätten, denen nicht entsprechende ärztliche Leistungen gegenüberstünden. Gesteuert werden würde das System in erster Linie vom »Bewertungsausschuss « auf Bundesebene als eine Art »ZK für Vergütung ", das sich auf der Grundlage kryptischer Expertise jeder demokratischen Kontrolle entzieht, meist auch der innerärztlichen. Das zeigt auch »Falk«, die neue Selbsthilfegruppe der KVen.

Dass ein liberaler Gesundheitsminister in die Gefahr gerät, der Verstaatlichung der ambulanten Versorgung die Hand zu reichen und jede, aber auch jede Vertragsfreiheit zu unterbinden, ist nicht ohne Ironie, wie dort ja auch ärztliche Praxen in der Trägerschaft einer öffentlich rechtlichen Körperschaft unter Mitgliedschaftspflicht liberaler erscheinen als in der Trägerschaft einer Kapitalgesellschaft, wobei die liberale $\mathrm{Sch}(\mathrm{m})$ erzgrenze irgendwo zwischen $\mathrm{GmbH}$ und Aktiengesellschaft liegt.

\section{Innovative Behandlungsmethoden}

An der Unterscheidung von »Erlaubnisvorbehalt « im ambulanten Sektor und »Verbotsvorbehalt « für die Einführung 
neuer Behandlungsmethoden im stationären Sektor soll offenbar festgehalten werden. Als ein erster Schritt im Sinne der notwendigen "kontrollierten « Einführung von Innovationen soll dem G-BA neben den bestehenden Alternativen (Anerkennung/Ausschluss/Aussetzung des Beschlusses) die Möglichkeit eröffnet werden, neue Untersuchungsund Behandlungsverfahren zeitlich begrenzt unter strukturierten Bedingungen zu erproben. Das Bewertungsverfahren soll in diesem Untersuchungszeitraum ausgesetzt werden. Die Finanzierung dieser Erprobungen soll, wie die Finanzierung des G-BA selbst, über den »Systemzuschlag « erfolgen, die Hersteller werden an der Finanzierung beteiligt. Die Generierung von Daten über den Nutzen einer neuen Methode erfordert in der Regel kontrollierte und möglichst randomisierte Studien. Solche Studien würden allein schon an der $\mathrm{Pa}$ tientenrekrutierung scheitern, wenn die neue Methode nicht zunächst auf die zur Erprobung im Rahmen der Studie ausgewählte Klinik beschränkt ist. Dadurch stiege im Übrigen die Patientensicherheit.

\section{Optionen der Krankenkassen auf der Leistungsseite}

Angesprochen werden ausdrücklich nicht Leistungen, die auf der Grenze zur PKV liegen. Der Eckpunkt testet offenbar den Widerstand in der Koalition aus, auf der Grundlage des bestehenden Leistungskataloges Teile in Satzungs- und Ermessensleistungen zu führen, die dann naturgemäß nicht mehr in den M-RSA einbezogen sind. Die Verweise auf Vereinbarungen mit Patientenorganisationen und Behindertenverbänden richten sich auf das niederländische Beispiel, bei dem der Leistungskatalog gesetzlich abgeschlossen worden ist und die GKV ebenso wie die PKV zusätzliche Leistungen anbieten können.
Fazit

Die Eckpunkte einer Reform sind ein politisches Zwischenergebnis, für das sich ein Fazit verbietet. Bilanz zu ziehen ist nach Veröffentlichung im Gesetzblatt. Aber sicherlich verspricht die Koalition mit dem Versorgungsgesetz eine relevante Reform und weithin notwendige Schritte. Die Chancen liegen im Wesentlichen bei der Verständigung mit den Ländern im Hinblick auf eine sektorübergreifende Bedarfsplanung, die auch die spezialärztliche Versorgung sinnvoll einbetten und dem Vertragswettbewerb einen Rahmen geben kann. Die Risiken liegen in einem sich selbst definierenden, autokratischen Versorgungssystem auf der ambulanten Seite, das Beitrags- und Steuerzahler zur Kassen bittet, ohne dass dem Leistungen für eine bessere medizinische Versorgung gegenüberstünden.

\title{
Neue Wege aus der impliziten Rationierung
}

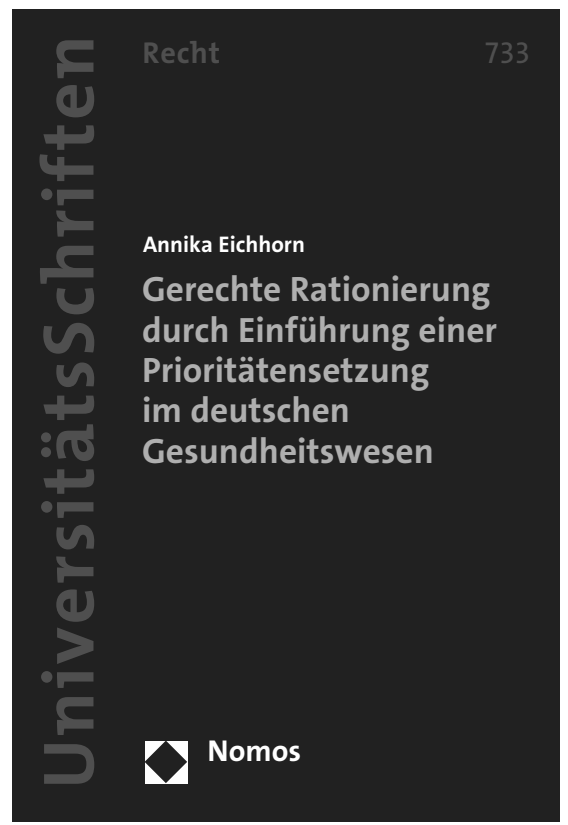

\author{
Gerechte Rationierung durch Einführung einer \\ Prioritätensetzung im deutschen Gesundheitswesen \\ Von Dr. Annika Eichhorn \\ 2011, ca. 250 S., brosch., ca. 64,-€, ISBN 978-3-8329-6641-6 \\ (Nomos Universitätsschriften - Recht, Bd. 733) \\ Erscheint ca. August 2011 \\ nomos-shop.de/13685
}

Die Ausschöpfung von Rationalisierungsreserven reicht nicht mehr aus, um den Mehrbedarf im deutschen Gesundheitswesen zu decken. Die Priorisierung in der Medizin begegnet den unausweichlich notwendigen Leistungseinschränkungen in transparenter und expliziter Weise unter Berücksichtigung ethischer, rechtlicher, ökonomischer und gesellschaftlicher Aspekte.

Bitte bestellen Sie im Buchhandel oder versandkostenfrei unter $\checkmark$ www.nomos-shop.de

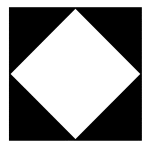

Nomos 